\title{
A Comprehensive Predictive Model to Anticipate Droplets Size Distribution in Pressure Swirl Atomizer
}

\author{
Sajad jafari ${ }^{1}$, Azadeh Kebriaee*1, Shahin sohrabi ${ }^{2}$ \\ ${ }^{1}$ Department of Aerospace engineering, Sharif University of Technology, Iran \\ ${ }^{2}$ Department of Mechanical engineering Universitat Politècnica de Catalunya, Spain \\ *Corresponding author: kebriaee@sharif.ir
}

\begin{abstract}
A predictive model is proposed to determine the droplet size and velocity distribution at the beginning of the secondary breakup. Liquid bulk is subjected to three stages of atomization including flow inside the injector, primary atomization, and secondary atomization to change a spray. The flow inside pressure swirl injector consisting an axial air core is a strongly complex two phase flow studied by highly precision coupled level set and volume of fluid method (CLSVOF). Subsequently, breakup lengths, interface deformation, as well as the most unstable frequency mode are calculated by nonlinear stability theory applied to emanating liquid sheet. Application of maximum entropy principle (MEP) is the final stage to predict size and velocity distribution of spray droplets. Based on liquid sheet breakup and conservative equations involving continuity, momentum, and energy, Newton-Raphson method is used to solve MEP equations. The proposed model has no dependency on empirical data in comparison with the usual past researches. The predicted results are shown to be in good agreement with the experimental findings related to performance characteristics and droplets size.
\end{abstract}

Keywords

Atomization, CLSVOF, Nonlinear Planar Liquid Sheet Stability, MEP, SMD.

\section{Introduction}

SPRAY modelling is a fundamental tool in the development of combustion chamber study. Fuel injectors have a key role on the combustion instability in gas turbine engines, internal combustion engines, and industrial furnaces [1]. Different correlations used to predict droplets size have no sufficient credit to apply in any arbitrary conditions. An objective procedure considering effective parameters, therefore, strongly helps designer in the parametric study. In the proposed method, representation of a comprehensive model for spray simulation leads to elicit the plenary information of injector performance with regarding the whole stages of atomization. Liquid bulk is subjected to different phases including flow inside the injector, primary atomization, and secondary atomization to change the spray droplets. Regardless of simple geometry of pressure swirl atomizer, the transition from a bulk of flow to the micron droplets is extremely complex. The atomization especially pressure swirl injector involves a transition twophase flow with recirculation region inside the injector and a complex unstable flow after issuing from injector [2]. The studies on injector are usually restricted to simulation and modelling a specific stage of atomization [3]. The numerical or experimental study on the flow inside the injector is the first step to determine macroscopic parameters of injector $[4,5]$. Primary Atomization attributed to liquid sheet breakup is the second stage of atomization. The transition from liquid bulk to ligaments and subsequently parent droplets is a strongly complex phenomenon. Two traditional methods are instability theory and breakup visualization to understand breakup mechanism $[6,7,8]$. The last stage in atomization is attributed to the secondary atomization. Final Sauter mean diameter, mean velocity, and uniformity of spray are the most important microscopic parameters usually studied experimentally [9, 10]. In the present work, we introduce a comprehensive model to predict the droplet size distribution. The two phase flow of axial air core inside the injector is simulated by coupled level set and volume of fluid method CLSVOF [11]. CLSVOF is a remedy to resolve the problems of level set and VOF methods corresponding to mass conservation and interface construction, respectively. Since extending the flow domain to outside of injector is a costly solution to identify the primary atomization of liquid sheet, a more inexpensive method should be introduced to determine the breakup procedure. The nonlinear hydrodynamic instability theory is applied to calculate the breakup length as well as interface deformation induced by the most unstable frequency [12]. The requirements of nonlinear instability theory are thickness of liquid sheet and velocity magnitude of film derived by numerical simulation of flow inside the injector. The calculation of size and velocity distribution of droplets is the last stage to determine injector performance. Maximum entropy principle (MEP) is a method to directly compute probability density function of droplets size and velocity $[13,14]$. What calculated is the spray information in the secondary atomization threshold and a designer can apply this straight forward procedure to predict injector behaviour. 


\section{Mathematical models and equations}

\subsection{Flow Simulation inside the Injector}

The tangential slots in pressure swirl injector causes to form an air core in the axial zone of the atomizer. One of the most current methods to simulate multi-phase flow is volume of fluid (VOF) [15]. The low storage requirement makes VOF a popular method to apply in numerical two phase flow simulations. In VOF, zero value is attributed to empty cell of fluid and subsequently a cell included the interface is evaluated by a value in a range of zero to one [16]. Besides wide applications of VOF in two phase flow simulations, the disadvantages of VOF involve the difficulty of interface reconstruction, the lack of adequate precision in the location of interface, and high dependency of solution accuracy to domain grid. So, Level Set (LS) method was developed as the VOF alternative by Osher [17]. The LS function is a signed function defined to specify the cell distance from the interface [18]. Based on LS function definition, the point on the interface is set to zero and the positive value is attributed to one fluid and negative to another. Unlike VOF, the interface location and its characteristics can be predicted accurately in LS method. In contrast, the continuity equation is not satisfied due to non-conservative inherent of LS function. A remedy to eliminate of disadvantages of these two-phase flow simulation methods is to introduce a hybrid solution. In the method of VOF and LS coupling, two methods are simultaneously solved to cover the problems [18]. In our first attempt, results were validated with experimental finding of Horvay and Leuckel [19]. The injector evaluates our simulation as figure.1.

\subsection{Instability in Planar Liquid Sheet Emanating from the Nozzle}

The first theoretical study on non-viscous liquid sheet instability was reported by Squire [20]. Following Squire, various studies on instability of round jet and sheet were performed with more accurate considerations either about assumptions on liquid and gas flow or methods of solution. For example, nonlinear instability analyses on sheet and jet were performed to understand more details about breakup mechanism. Following, two-dimensional inviscid and incompressible liquid sheet with $2 a^{*}$ thickness is schematically shown in Fig.2, where the liquid sheet with axial uniform velocity of $U_{l}$ moves through the gaseous medium [12]. When the basic non-rotational flow is perturbed by small disturbances, the liquid-gas interfaces are shifted to the new location with $y^{*}=(-1)^{j+1}+\eta_{j}$ coordinates. Where $j=1$ indicates the top and $j=2$ the bottom interface. Based on none-dimensional velocity potentials, the continuity equations of liquid and gas phases are defined as equations $(1,2)$.

$$
\begin{array}{llc}
\phi_{l, x x}+\phi_{l, y \mathrm{y}}=0 & \text { for } & -1+\eta_{2}<y<1+\eta_{1} \\
\phi_{\mathrm{g}, x x}+\phi_{\mathrm{g}, \mathrm{yy}}=0 & \text { for } & 1+\eta_{1}<y<\infty
\end{array}
$$
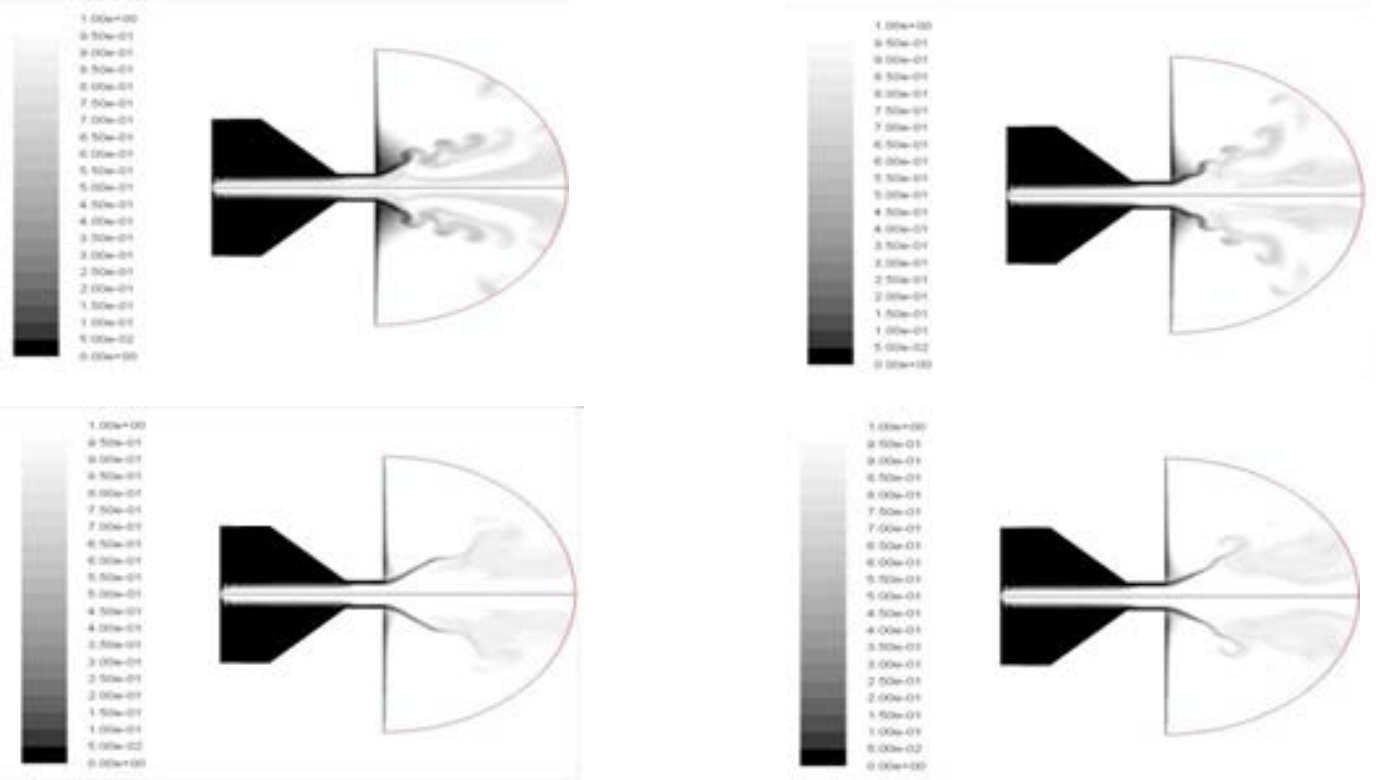

Fig 1. Numerical simulation by CLSVOF code for capturing of the interfaces for Horvay \& Leuckel's injector 
Where length, time, and density are normalized by half thickness of $a^{*}$, convection time and liquid density, respectively. In addition, the interfacial boundary condition is derived as,

$$
v_{i}=\frac{D \eta_{i}}{D t}=\frac{d \eta_{i}}{d t}+U_{i} \frac{d \eta_{i}}{d x}
$$

Where $U_{i}=\frac{d \phi}{d x}$ and $v_{i}=\frac{d \phi}{d y}$ are velocity components and the kinematic boundary conditions are simplified as,

$$
\begin{aligned}
& \phi_{l, y}-\eta_{j, t}-\phi_{l, \mathrm{x}} \eta_{j, \mathrm{x}}=0 \\
& \phi_{\mathrm{g}, y}-\eta_{j, t}-\phi_{\mathrm{g}, \mathrm{x}} \eta_{j, \mathrm{x}}=0
\end{aligned}
$$

The force balance at the interface leads to the dynamic boundary conditions as,

$$
\frac{1}{2}-\frac{1}{2} \rho U^{2}+\rho \phi_{g, t}-\phi_{l, t}+\frac{1}{2} \rho\left(\phi_{g, x}^{2}+\phi_{g, y}^{2}\right)-\frac{1}{2}\left(\phi_{l, x}^{2}+\phi_{l, y}^{2}\right)=\frac{(-1)^{j} \eta_{j, x x}}{W e\left(1+\eta_{j, x}{ }^{2}\right)^{\frac{3}{2}}}
$$

Applying regular perturbations method to expand the interface displacement, the following forms can be assumed to specify the first and second order surface deformations, respectively,

$$
\begin{aligned}
& \eta_{j, 1}(x, t)=A_{1, s} \cosh \left(\omega_{1, s} t\right) \cos \left(\alpha_{1, s} t+k x\right) \\
& \eta_{j, 2}(x, t)=p_{2, s}(t) \cos (2 k x)+Q_{2, s}(t) \sin (2 k x)
\end{aligned}
$$

Where $\omega_{1, s}$ and $\alpha_{1, s}$ are the growth rate and angular frequency, respectively, and $\mathrm{A}_{1, \mathrm{~s}}$ is the amplitude of disturbed deformation. After sorting governing equations as well as kinematic and dynamic boundary conditions in terms of first order and second order in terms of $\eta_{0}$, we have the equations of (10-20). By simplifying the calculations based on the coefficient of sinus and cosine for the first and second order of surface deformations, respectfully the equations are solved. After tedious mathematical manipulations, the growth rate of surface deformation can be determined in the eigenvalue equation as equation (15). The variations of growth rate vs. wave number are shown in Fig 3 for the case of $\rho=0.001, U=4, W e=40$. As seen, $\omega_{1, s}$ has a peak in terms of wave number, corresponding to the most unstable mode of liquid sheet breakup. According to Fig 3 , the most unstable frequency is related to $k=0.181$ wave number for the first mode of instability discussed in [12]. In Fig.4, the growths of interface deformation in different times are depicted for $\rho=0.001, U=4, W e=40$, and $k=0.181$. Fig 4 prescribes the temporal interface disturbance growth and consequently sheet breakup occurs about $t \approx 145$. Based on Gaster's transformation [21], Fig 5 represents the locus of interfaces deformation for the case of $\rho=0.001$, $U=4, W e=40$.

$$
\begin{aligned}
& \eta_{j}(\mathrm{x}, \mathrm{t})=\sum_{n=1}^{\infty} \eta_{0}^{n} \eta_{j, n}(\mathrm{x}, \mathrm{t}) \\
& \phi_{l 1, x x}+\phi_{l 1, y \mathrm{y}}=0 \text { for }-1<y<1 \\
& \phi_{\mathrm{g} 1, x x}+\phi_{\mathrm{g} 1, \mathrm{yy}}=0 \text { for }+1<y<+\infty,-\infty<y<-1 \\
& \phi_{l 1, y}-\eta_{j, 1, t}-\eta_{j, 1, \mathrm{x}}=0 \\
& \phi_{l 1, y}-\eta_{j, 1, t}-\eta_{j, 1, \mathrm{x}}=0 \\
& \rho \phi_{g 1, x}+\phi_{l 1, \mathrm{t}}+\rho U \phi_{g 1, x}-\phi_{l 1, x}-\frac{(-1)^{j}}{w e} \eta_{j, 1, x x}=0 \\
& k \sqrt{\tanh (k) \rho(U-1)^{2}-k \frac{\rho+\tanh (k)}{W e}}
\end{aligned}
$$

\section{First order of $\eta_{0}$}




$$
\begin{aligned}
& \phi_{12, x x}+\phi_{12, y \mathrm{y}}=0 \text { for }-1<y<1 \\
& \phi_{12, y}-\eta_{j, 2, \mathrm{t}}-\eta_{j, 2, \mathrm{x}}=\eta_{j, 1, \mathrm{x}} \phi_{11, x}-\eta_{j, 1} \phi_{11, \mathrm{yy}} \\
& \phi_{12, y}-\eta_{j, 2, \mathrm{t}}-\eta_{j, 2, \mathrm{x}}=\eta_{j, 1, \mathrm{x}} \phi_{11, x}-\eta_{j, 1} \phi \\
& \phi_{g 2, y}-\eta_{j, 2, \mathrm{t}}-U \eta_{j, 2, \mathrm{x}}=\eta_{j, 1, \mathrm{x}} \phi_{g 1, x}-\eta_{j, 1} \phi_{g 1, \mathrm{yy}} \\
& \phi_{g 2, \mathrm{t}}-\phi_{12, \mathrm{t}}+\rho U \phi_{g 2, x}-\phi_{12, x}-(-1)^{j} \frac{\eta_{j, 2, x x}}{w e}=-\rho\left[\eta_{j, 1, \mathrm{t}} \phi_{g 1, y}+\eta_{j, 1} \phi_{g 1, y t}\right] \\
& +\left[\eta_{j, 1, \mathrm{t}} \phi_{11, y}+\eta_{j, 1} \phi_{11, y t}\right]-\frac{1}{2} \rho\left[\phi_{g 1, x}^{2}+\phi_{g 1, y}^{2}+2 \mathrm{U}\left(\eta_{j, 1, \mathrm{x}} \phi_{g 1, y}+\eta_{j, 1} \phi_{g 1, y x}\right)\right]+\frac{1}{2}\left[\phi_{11, x}^{2}+\phi_{1, y}^{2}+2\left(\eta_{j, 1, x} \phi_{11, y}+\eta_{j, 1} \phi_{11, y x}\right)\right]
\end{aligned}
$$

\subsection{Velocity and Droplet Size Distribution in Spray}

The physics beyond the nozzle flow and sheet breakup are deterministic concepts [2], while spray atomization is a perfectly stochastic process with different size and velocity droplets $[22,23]$. As known, the probability density function (PDF) of droplets size and velocity is introduced to define spray characteristics. A solution to confront with difficulty of spray study is to use the Shannon maximum entropy principle (MEP), independent of experimental primary data [23]. This method was introduced by [13] and later developed by Li and Takin [24]. In addition, recent investigations attributed to Movahednejad have improved the modified momentum equations in MEP [25]. Shannon stated the form of entropy as, $[23,25]$

$$
s=-\kappa \sum f \ln f
$$

Where $\mathbf{s}$ is the statement of statistical thermodynamic entropy $\kappa$ is the Boltzmann's constant and $\mathbf{f}$ is the probability density function. Besides to establish the maximum condition of thermodynamic entropy, the conservation equations including mass, momentum, and energy should be satisfied in sheet breakup. Supposed spherical droplets, the conservation equations are introduced based on PDF as equations (22-26)

$$
\begin{aligned}
& \int_{\bar{D}_{\min }}^{\bar{D}_{\max }} \int_{\bar{u}_{\min }}^{\bar{u}_{\max }} f \bar{D}^{3} d \bar{D} d \bar{u}=1+\bar{s}_{m} \\
& \int_{\bar{D}_{\max }}^{\bar{D}_{\max }} \int_{\bar{u}_{\min }}^{\bar{u}_{\min }} f \bar{D}^{3} \bar{u} d \bar{D} d \bar{u}=1+\bar{s}_{m u} \\
& \int_{\bar{D}_{\min }}^{\bar{D}_{\max }} \int_{\bar{u}_{\min }} f\left(\frac{\bar{D}^{3} \bar{u}^{2}}{H}+\frac{B \bar{D}^{2}}{H}\right) d \bar{D} d \bar{u}=1+\bar{s}_{e}
\end{aligned}
$$

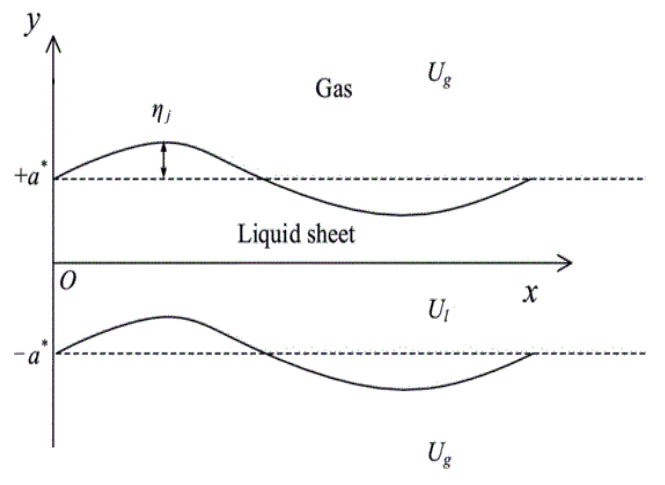

Figure 2. Planar liquid sheet [1]



Figure 3. Instability growth rate vs. wave number for $\rho=0.001$,

$$
U=4, W e=40
$$



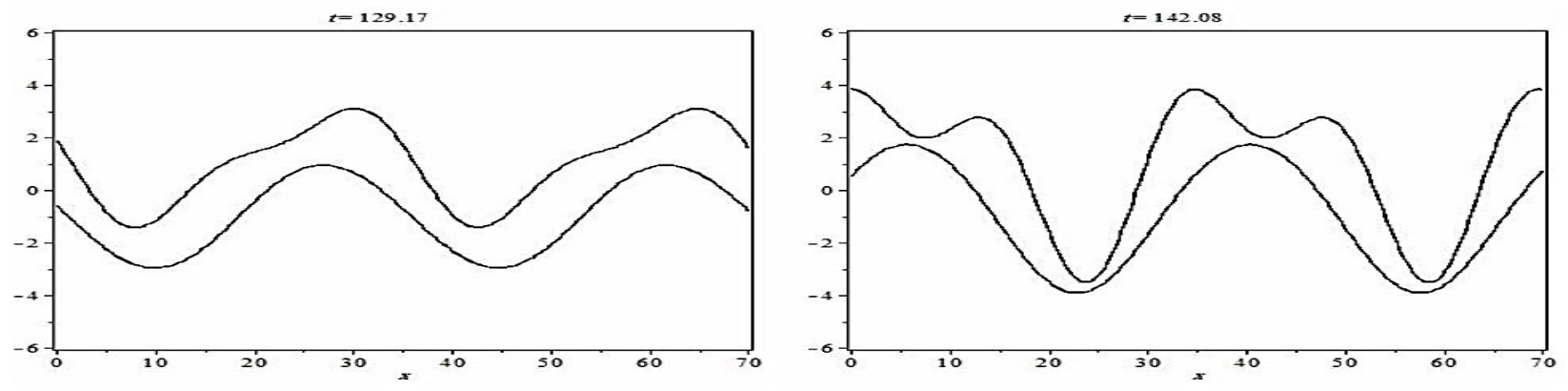

Fig 4. Temporal surface deformation evolution for $\rho=0.001, U=4, W e=40$, and $k=0.181$

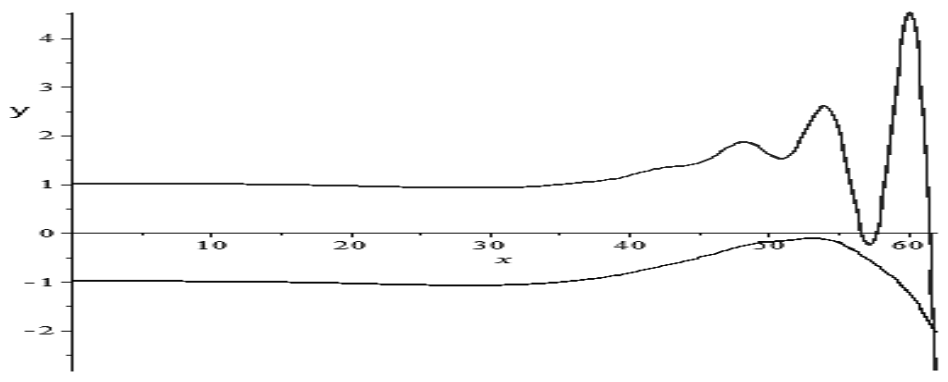

Fig 5. How breakup of the liquid film after issuing from the nozzle

$$
B=\frac{12}{W e}, W e=\frac{\rho_{l} U_{l}^{2} D_{30}}{\sigma}
$$

According PDF concept, the following equation is also introduced,

$$
\int_{\bar{D}_{\min }}^{\bar{D}_{\max }} \int_{\bar{u}_{\min }}^{\bar{u}_{\max }} f \bar{D}^{3} d \bar{D} d \bar{u}=1
$$

It is worthy to note that Eqs. (22-24) and (26) are non-dimensionlaized by $D_{30}$ and $U_{l}$ known as mean diameter and the potential velocity of liquid sheet. Also, the probability density function using Lagrange multipliers method can be obtained as equation (27).

$$
f=f_{0} \exp \left[-\lambda_{0}-\lambda_{1} \bar{D}^{3}-\lambda_{2} \bar{D}^{3} \bar{u}-\frac{\lambda_{3}}{H}\left(\bar{D}^{3} \bar{u}^{2}+B \bar{D}^{2}\right)\right]
$$

If the velocity profile at the injector outlet is assumed uniform, the shape factor of velocity profile $H$ will be unity. But if the outlet flow from the injector is assumed to be fully developed and turbulent, this factor will be equal to [25, 26]. The accuracy of this formulation is confirmed with comparing with experimental work of Li [27].

\section{RESULTS \& DISCUSION}

As mentioned, following different stages of atomization will be conducted to determine the velocity and droplet size distribution at the secondary atomization threshold. The prediction of droplet size and velocity distribution is categorized in three stages including flow inside the injector, sheet instability near the nozzle, and primary atomization. The interaction of air core and highly swirling flow inside the injector is simulated by CLSVOF Capturing accurate location and geometry of air core. Two key findings of flow simulation inside the injector are liquid film thickness and liquid velocity used to calculate the sheet breakup length in nonlinear instability theory. Extending instability theory to nonlinear terms causes directly to predict deformation topology of sheet in the breakup of moment. At the last stage, the droplets velocity and size distribution are calculated by solving MEP equations. The probability density function of size and velocity is a comprehensive map to derive all of spray characteristics included mean diameter, Sauter mean diameter, mean velocity as well as the parameters of RosinRammler function to specify droplet size distribution [2]. 

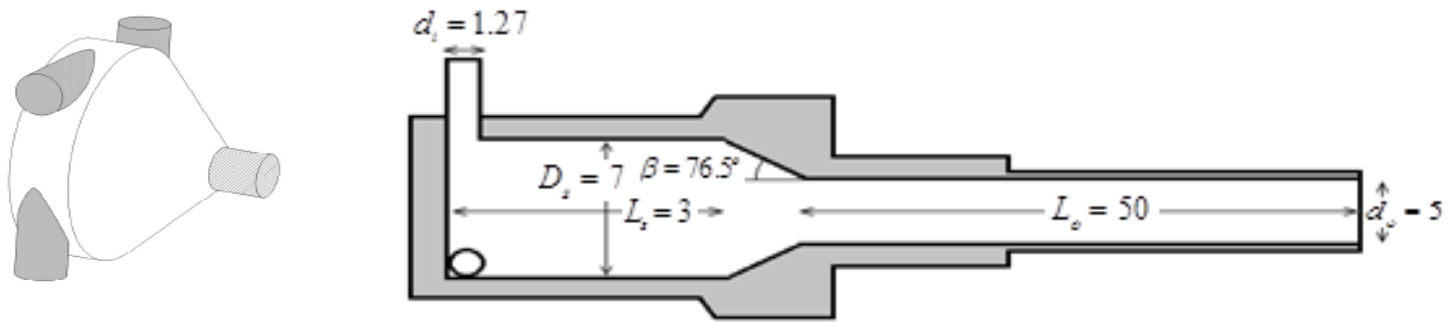

Fig 6. Geometrical schematic of injector [28]

In this paper, the results of Eberhart's experimental results were used to evaluate the proposed comprehensive model. Eberhat examined a pressure swirl injector to study spray angle, liquid film thickness, and droplets size by PDPA method, at different flow rates [28]. The geometrical schematic of injector has been illustrated in Fig 6 and injector dimensions are numerically shown in the figure in millimetre [28]. The case with mass flow rate $2.809 \times 10^{-3} \mathrm{~kg} / \mathrm{s}$ was chosen to simulate. As mentioned, CLSVOF was used to model two phase flow field of injector by 110000 structural grid cells after validating mesh dependency [4]. The method of RANS equations solution has been completely demonstrated in and the validation of solution was confirmed based on experimental results of [19]. Fig 7 illustrates how the air core is formed in the axial zone by representation of volume of water inside the injector. Attenuation of liquid sheet is seen due to accelerating axial velocity in orifice part by decreasing cross section of flow. The recirculating flow near the nozzle represents positive pressure gradient caused by free vortex flow inside the injector. The results in Fig 8 confirm the last claim about pressure distribution. As seen, free vortex flow leads to increase of swirl velocity near the axis and subsequently decrease of pressure in the whole length of injector. The numerical results of injector performance characteristics included injector discharge coefficient, liquid film thickness, and spray half angle are quantitatively compared with experimental results. Results describe the maximum error in the numerical simulation of injector is less than $13 \%$ to determine performance characteristics inside the injector. The most unstable wave number of liquid sheet is $k=1.158$ based on Eq. (10), corresponding to $\rho=0.001, U=0$, and $W e=1913$.

Where the magnitude velocity of flow at the exit of injector $U_{l}=28$ and half thickness of liquid sheet $2 a^{*}=0.41 \mathrm{~mm}$ are applied to non-dimensionalize the nonlinear instability equations. It is worthy to note that nonlinear instability analysis (NLISA) is an alternative of LISA (Linear Instability Analysis) for more accurate location of issuing the primary droplets in the computational flow field. In Fig 9, the shape of liquid sheet is depicted for $\eta_{0}=0.01$, and consequently the length of breakup is predicted about $23 \mathrm{~mm}$ in NLISA. The experimental breakup length equals $26 \mathrm{~mm}$ has been compared with NLISA. The more precise prediction of breakup in NLISA is obviously seen by quantitative calculation.

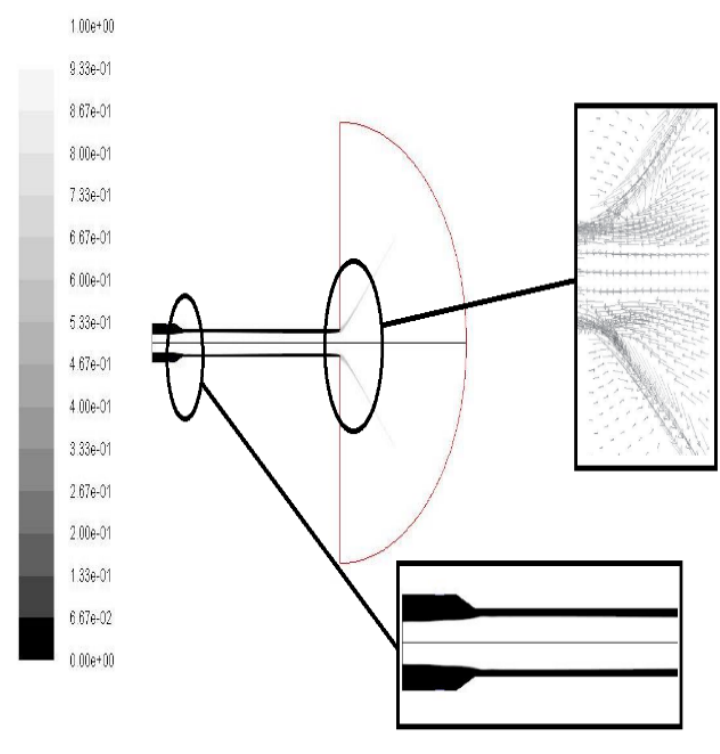

Fig 7. Contour of water volume in pressure swirl injector

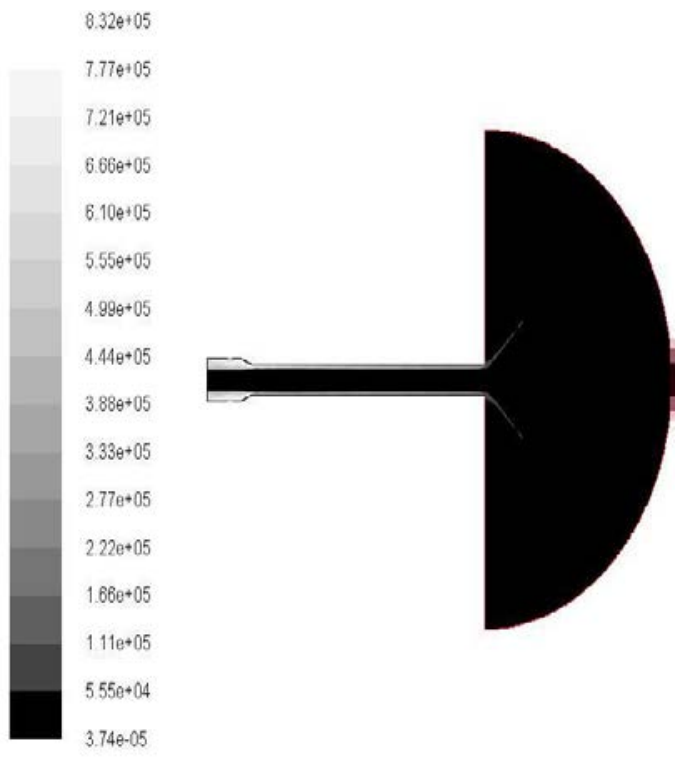

Fig 8. Contour of pressure in the flow domain 


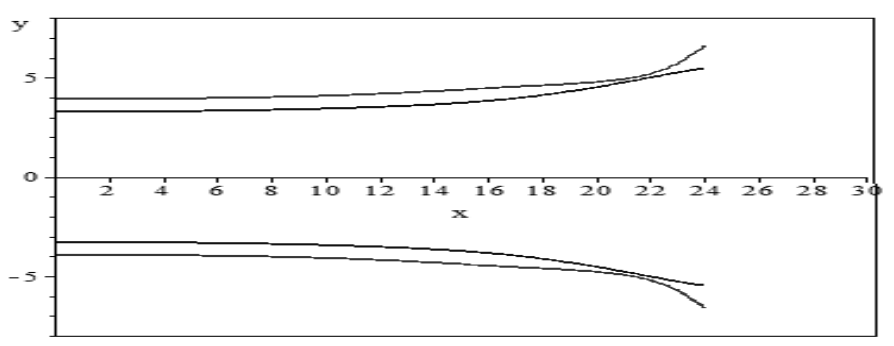

Fig 9. Liquid interfaces simulation by nonlinear instability theory

The droplets velocity and size distribution for current flow are achieved by solving MEP equations. After calculating Lagrange's multipliers, PDF of droplets size $(d)$ and velocity $(u)$ are represented in Fig 10. PDF includes the whole information of spray characteristics. For example, the Sauter mean diameter (SMD) is numerically integrated by following equation.

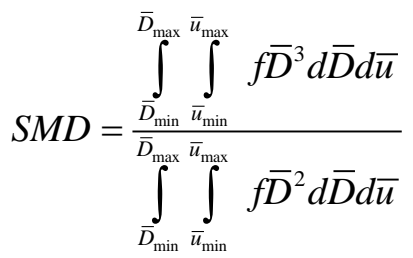

Based on PDF calculated by MEP equations, Sauter mean diameter is calculated about $80 \mu \mathrm{m}$, reported about $100 \mu \mathrm{m}$ in experiment, [28]. Although the results depict an error about $20 \%$ in this calculation, it should be noted this is an accumulated error in different simulation stages. It should be noted that Sauter mean diameter is not a unique parameter to validate the solution and it may be some other parameters not reported in literature is completely near the simulation results like mean diameter or average velocity of droplets.

\section{Conclusion}

A comprehensive model is a unique solution to predict the injector performance in spray production. The tangential slot embedded on the injector body leads to a free vortex flow inside the injector causing air core in the axial zone. The complex two phase flow in the small size of the injector necessitates application of CLSVOF to determine thickness of liquid film, tangential and axial velocity in the injector nozzle. The output of flow simulation inside the injector is used in calculation of planar hydrodynamic instability to determine the manner of breakup process. The knowledge about the most unstable frequency calculated in this part helps to predict the size and velocity distribution function in spray. Probability density function of size and velocity in spray is computed by maximum entropy principle method. In MEP method, the maximum entropy is applied as a supplementary constraint to introduce a unique function of size and velocity distribution of droplets. Newton-Raphson is used to solve conservative governing equations. After calculating Lagrange's multipliers, PDF is determined, and subsequently Sauter mean diameter is calculated. The acceptable results compared with experiments guaranty the idea of this model to predict macroscopic as well as microscopic parameters of injector.

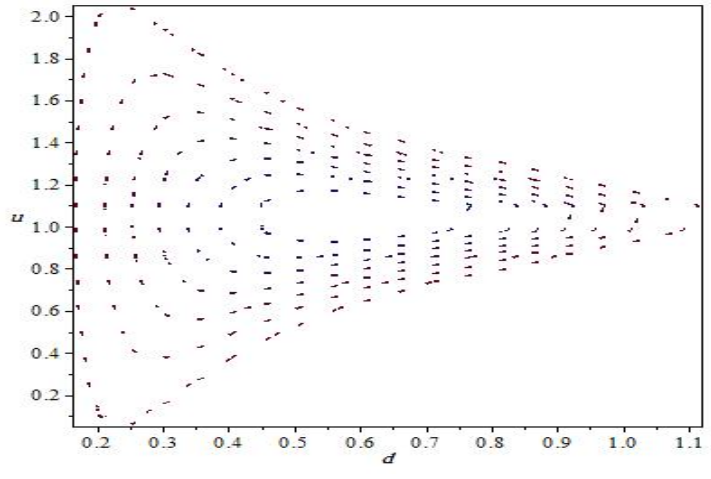

A) Two-dimensional contour

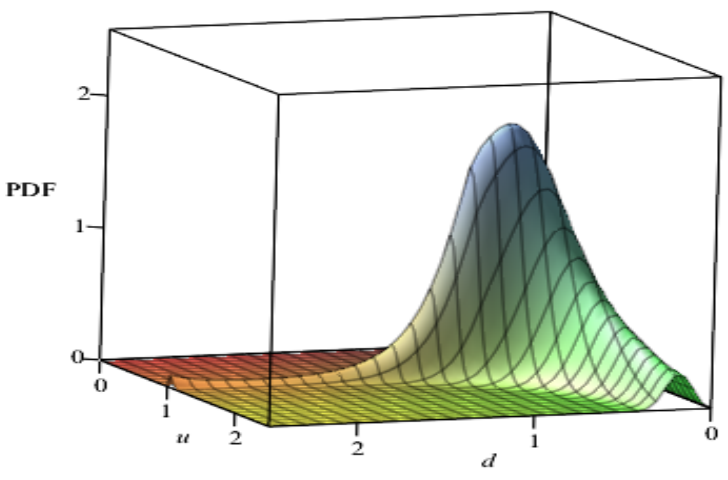

B) Three-dimensional contour

Fig 10. PDF of droplets size and velocity 


\section{REFERENCES}

[1] Ashgriz N. ed. (2011), "In Handbook of Atomization and Sprays", Springer, 339-358.

[2] Lefebvre A. H. (1989), "Atomization and Sprays, Hemisphere", Bristol Pa, U.S.A.

[3] Tharakan, T., Mukhopadhyay, A., Datta, A., and Jog, M. (2013), "Trends in Comprehensive Modeling of Spray Formation," International Journal of Spray and Combustion Dynamics, vol. 5, no. 2, pp. $123-180$.

[4] Nouri-Borujerdi, A., and Kebriaee, A. (2012). "Numerical Simulation of Laminar and Turbulent Two-Phase Flow in Pressure-Swirl Atomizers", AIAA Journal, Vol. 50, No. 10, pp. 2091-2101.

[5] Shafaee, M., Banitabaei, S. A., Esfahanian, V., Ashjaee, M.(2012), "An investigation on effect of geometrical parameters on spray cone angle and droplet size distribution of a two-fluid atomizer", Journal of Mechanical Science and Technology, vol. 25, No. 12, pp. 3047-3052.

[6] Ibrahim, A. A., Jog, M. A.(2003), "Effect of liquid and air swirl strength and relative rotational direction on the instability of an annular liquid sheet ",Acta Mechanica, vol. 186, no. 1, pp. 113-133.

[7] Lad, V. N., Murthy, Z. V. P.(2016)," Effects of the geometric orientations of the nozzle exit on the breakup of free liquid jet", Journal of Mechanical Science and Technology, vol. 30, no. 4, pp. 1625-1630.

[8] Inamura, T., Tamura, H. \& Sakamoto, H.(2003), "Characteristics of Liquid Film and Spray Injected from Swirl Coaxial Injector," Journal of Propulsion and Power, vol. 19, no. 4, pp. 632-639.

[9] T. Marchione, C. Allouis, A. Amoresano, and Federico Beretta(2007), "Experimental Investigation of a Pressure Swirl Atomizer Spray", Journal of Propulsion and Power, Vol. 23, No. 5, pp. 1096-1101.

[10] Shim, Y. S., Choi, G. M., Kim, D. J. (2008), "Numerical and Experimental Study on Hollow-Cone Fuel Spray of High Pressure Swirl Injector under High Ambient Pressure Condition", Journal of Mechanical Science and Technology, vol. 22, no. 2, pp. 320-329.

[11] Sussman, M., Puckett E. G. (2000), "A Coupled Level Set and Volume-of-Fluid Method for Computing 3D and Axisymmetric Incompressible Two-Phase Flows", Journal of Computational Physics, vol. 162, No. 2, pp. $301-337$.

[12] Mitra, S.K. (2001), "Breakup Process of Plane Liquid Sheets and Prediction of Initial Droplet Size and Velocity Distribution in Sprays," Ph. D Thesis, University of Waterloo.

[13] Sellens R.W., Brzustowski T. A.(1985), "A Prediction of Drop Size Distribution in a Spray from Principle", Atomization and Spray Technology, 1, 89-102.

[14] Sellens R.W., Brzustowski T.A. (1986), "A Simplified Prediction of Droplet Velocity Distribution in a Spray", Combustion and Flame, 65, 273-279.

[15] Xue, J. (2004), "Computational Simulation of Flow inside Pressure Swirl Atomizers", Ph. D. Thesis, University of Cincinnati.

[16] Yeh, Ch. L. (2007), "Numerical Simulation of a Turbulent Liquid Jet Emanating from a Plain-Orifice Atomizer and a Pressure-Swirl Atomizer", Numerical Heat Transfer Applications, vol. 51, no. 12, pp. 1187-1212.

[17] Osher, S. and Sethian, J.A. (1988), "Fronts propagating with curvature-dependent speed: Algorithms based on Hamilton-Jacobi formulations," Journal of Computational Physics, vol. 79, no. 1, pp. 12-49.

[18] Sussman M., Fatemi E., Smereka P., Osher S.(1998), "An improved level set method for incompressible two-phase flows," Computers \& Fluids, vol. 27, no. 5-6, pp. 663-680.

[19] Horvay M., Leuckel W.(1986), "Experimental and Theoretical Investigation of Swirl Nozzles for Pressure-Jet Atomization", German Chemical Engineering, 5, 276-283.

[20] Squire, H.B. (1953), "Investigation of the instability of a moving liquid film", British Journal of Applied Physics, vol. 4 , no. 6, pp. 167-169.

[21] Gaster M. (1962),"A note on the relation between temporally-increasing and spatially-increasing disturbances in hydrodynamic stability," Journal of Fluid Mechanics, vol. 14, no. 02, p. 222

[22] Dumouchel C. (2006), "A New Formulation of the Maximum Entropy Formalism to Model Liquid Spray Drop-Size Distribution", Particle \& Particle Systems Characterization, vol. 23, no. 6, pp. 468-479.

[23] Dumouchel C. (2009), "The Maximum Entropy Formalism and the Prediction of Liquid Spray Drop-Size Distribution", Journal of Entropy, 11, 713-747.

[24] Li X., Tankin R.S. (1987), "Droplet size distribution: A Deviation of a Nukiyama-Tanasawa Type Distribution Function", Combustion Science and Tech., 56, 65-76.

[25] Movahednejad E., Ommi F., Hosseinalipour S. M., Chen .C. P., Mahdavi S. A.(2011), "Application of maximum entropy method for droplet size distribution prediction using instability analysis of liquid sheet," Heat Mass Transfer, vol. 47, no. 12, pp. 1591-1600.

[26] Mahdavi S.A., Movahednejad E., Ommi F., Hosseinalipour S.M.(2013), "Estimation of the Breakup Length for the Annular and the Round Liquid Jet Using Linear Instability Analysis", IJST, Transactions of Mechanical Engineering, 37, No. M2, 217-232.

[27] Li, X.; Chin, L.P., Tankin, R.S.; Jackson, T.; Stutrud, J., Switzer, G.(1991)“Comparison between experiments and predictions based on maximum entropy for sprays from a pressure atomizer," Combustion and Flame, vol. 86, no. 1-2, pp. 73-89.

[28] Eberhart, C. J, Lineberry, D. M. and Moser, M. D. (2009),“Experimental Cold Flow Characterization of a Swirl Coaxial Injector Element," 45th AIAA/ASME/SAE/ASEE Joint Propulsion Conference \&amp; Exhibit. 